\title{
Laser ablation in CdZnTe crystal due to thermal self-focusing: Secondary phase hydrodynamic expansion
}

\author{
A. Medvid’a,*, A. Mychko a , E. Dauksta ${ }^{\mathrm{a}}$, V. Kosyak ${ }^{\mathrm{b}}$, L. Grase $^{\mathrm{a}}$ \\ a Riga Technical University, 3 Paula Valdena Str., LV-1048 Riga, Latvia \\ b Sumy State University, 2, Rymskogo-Korsakova St., 40007 Sumy, Ukraine
}

\section{A R T I C L E I N F O}

\section{Article history:}

Received 22 June 2015

Received in revised form

17 September 2015

Accepted 27 September 2015

Available online $\mathrm{xxx}$

Keywords:

CdZnTe crystal

Laser ablation

Raman spectroscopy

Self-focusing

Te inclusions

\begin{abstract}
A B S T R A C T
The present paper deals with the laser ablation in CdZnTe crystal irradiated by pulsed infrared laser. Two values of threshold intensities of the laser ablation were determined, namely of about 8.5 and $6.2 \mathrm{MW} / \mathrm{cm}^{2}$ for the incident and the rear surfaces, correspondingly. Lower intensity of the laser ablation for the rear surface is explained by thermal self-focusing of the laser beam in the CdZnTe crystal due to heating of Te inclusions with a following hydrodynamic expansion.
\end{abstract}

(C) 2015 Elsevier B.V. All rights reserved.

\section{Introduction}

The CdZnTe ternary compound is widely used in a variety of microelectronics devices such as radiation detectors [1], solar cells [2] and infrared (IR) detectors [3,4]. However, CdZnTe radiation detectors made from as-grown crystals with a high concentration of point and extended defects, such as grain boundaries, twins, secondary phases (such as Te inclusions), are limited in their performance. In most cases, in order to improve the properties of CdZnTe crystal, the thermal annealing under $\mathrm{Cd}$ and Te atmosphere is used, which leads to changes in chemical composition and dopant redistribution in the whole sample [5]. Instead of the thermal annealing the pulsed laser annealing [6,7] provides a highly localized annealing effect. The irradiation of CdZnTe semiconductor crystal with nanosecond laser pulses has been used for the modification of structural, electrical and optical properties, as well as to induce changes in chemical composition [8-11].

In general, the interaction of the laser radiation with semiconductors is usually described by the thermal model [12]. In the case when nanosecond pulsed laser radiation is strongly absorbed by the material the thermal model is also used, taking into account the high temperature gradient at the surface, the socalled thermogradient effect [10]. In the case of low-absorbed IR

\footnotetext{
* Corresponding author.

E-mail address: mychko@latnet.lv (A. Medvid').
}

laser radiation, the uniform temperature field is formed in the bulk of the semiconductor due to uniform absorption of laser radiation.

In our recent study the possibility to improve parameters of radiation detector based on CdZnTe:In crystal with the help of IR laser radiation was shown [13]. This effect was explained by the precipitation of uncontrollable impurities by Te inclusions due to gradient temperature field around them and the increase in solubility of In atoms in CdZnTe:In crystal. However, to avoid damages of crystal surface it is necessary to determine the threshold intensity of laser ablation.

Therefore, the aim of this study is to determine the threshold intensities of laser ablation in the CdZnTe crystal.

\section{Experimental}

The $\mathrm{Cd}_{x-1} \mathrm{Zn}_{x} \mathrm{Te}(x=0.1)$ indium doped (3\%) single crystal has been grown by vertical gradient freeze method using high-purity $7 \mathrm{~N}$ materials.

The sample was cut from ingot, the surface of the sample was mechanically polished using $3 \mu \mathrm{m}$ and $1 \mu \mathrm{m}$ alumina oxide powder, and then by the diamond pastes with a size of an abrasive grains down to $0.1 \mu \mathrm{m}$. The dimension of the sample was $10.0 \mathrm{~mm} \times 10.0 \mathrm{~mm} \times 3.0 \mathrm{~mm}$. In order to produce smooth and clean defect-free surfaces the crystal was chemically etched by bromine-methanol solution, which is typically used for this purpose [14]. 


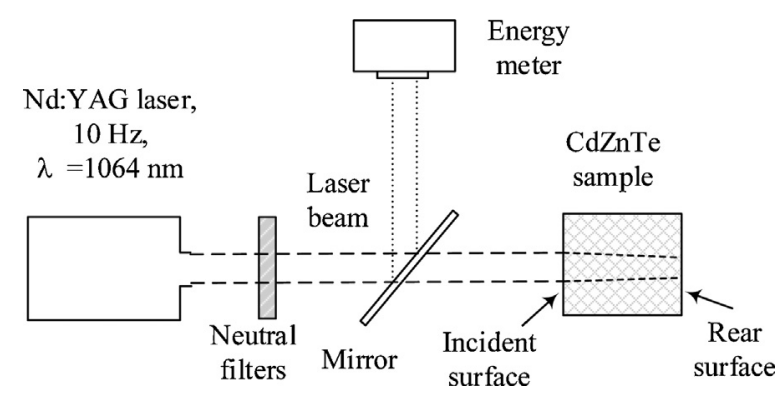

Fig. 1. Scheme of the experimental setup for laser annealing.

Before the laser processing the distribution and dimensions of Te inclusions was analyzed by the Nikon Eclipse LV150 optical microscope operated in IR mode.

The scheme of the experimental setup, which was used for laser annealing, is shown in Fig. 1. The CdZnTe crystal was irradiated by the Nd:YAG laser pulses at room temperature in air under normal atmospheric pressure. The laser operated in Q-switched mode with pulse duration of $5 \mathrm{~ns}$, power $1.0 \mathrm{MW}$, pulse repetition rate $10 \mathrm{~Hz}$, spot diameter $6 \mathrm{~mm}$, at a fundamental frequency with wavelength $1.064 \mu \mathrm{m}$. The position of the laser spot was not changed during irradiation. The incident laser radiation intensity was varied by neutral filters. The energy of laser pulse was measured by FieldMax II energy meter.

The micro-Raman spectroscopy is widely used for the analysis of CdZnTe phase composition [15-17]. In particular, this method allows to study distribution of Te inclusions [18-20] on a surface. In our study, Raman spectra were measured at room temperature by the Renishaw InVia micro-Raman spectrometer in a backscattering geometry using $1800 \mathrm{~mm}^{-1}$ grating for the $\operatorname{Ar}(514 \mathrm{~nm})$ laser excitation source. The resulting signal was collected by CCD camera. The calibration of the spectrometer was done by measuring Raman spectrum of a silicon wafer.

FEI Nova NanoSEM 650 Schottky field emission scanning electron microscope (SEM) was used for the study of CdZnTe crystal surface morphology after irradiation by the laser.

\section{Results and discussion}

As can be seen from Fig. 2a, initially, the surface of the sample had a high concentration of Te inclusions of $1.8 \times 10^{8} \mathrm{~cm}^{-2}$, with size distribution from 1 to $5 \mu \mathrm{m}$.

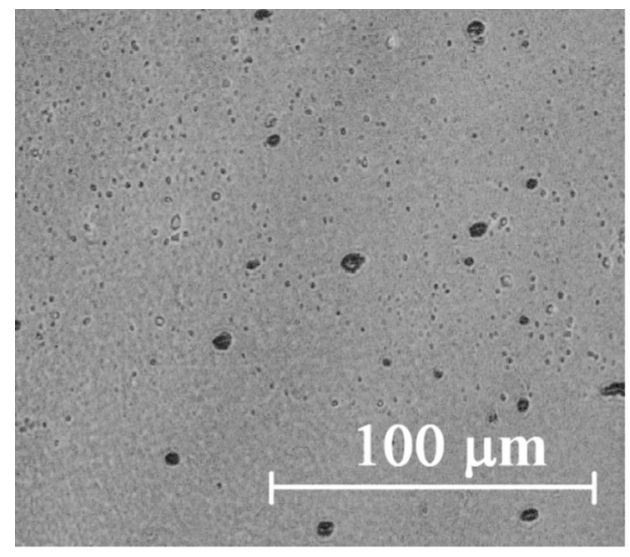

a)

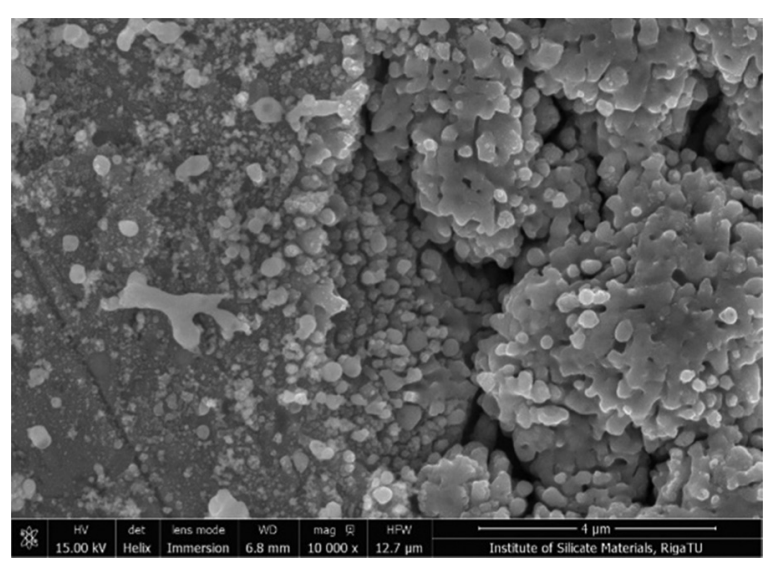

Fig. 3. SEM image of the rear surface after irradiation by the laser with intensity of $6.2 \mathrm{MW} / \mathrm{cm}^{2}$ for $0.5 \mathrm{~h}$.

The morphology of the incident surface was not changed after the irradiation by the laser with intensity of $6.2 \mathrm{MW} / \mathrm{cm}^{2}$ but the rear surface became porous with about the same surface concentration of micro-pores as Te inclusions (Fig. 2b). It was determined that the threshold intensity of the laser radiation for the incident surface was about $8.5 \mathrm{MW} / \mathrm{cm}^{2}$. Thus, we found that the threshold intensity for the incident surface is higher than for the rear surface.

We suppose that laser ablation of the rear surface is connected with the presence of Te inclusions. As was shown in our previous investigation [13] the laser radiation with energy of quantum $h \nu=1.1 \mathrm{eV}(\lambda=1.064 \mu \mathrm{m})$ is absorbed mainly by the Te inclusions, because the band gap of Te is $E_{\mathrm{g}}=0.33 \mathrm{eV}$. Whereas, the CdZnTe crystal with the band gap of $1.6 \mathrm{eV}$ is transparent for this radiation.

Our estimation according to the heat-flow equation [21] shows that the heating of CdZnTe does not occur due to the low absorption coefficient of $\sim 0.5 \mathrm{~cm}^{-1}$ for the wavelength of $1.064 \mu \mathrm{m}$, even if the two-photon absorption is taken into account. As a result at this laser intensity only Te inclusions are heated close to melting temperature of $449^{\circ} \mathrm{C}$, which is about 2 times lower than the melting temperature of $\mathrm{CdZnTe}$ which is about $900^{\circ} \mathrm{C}$. Hence due to the low thermal conductivity of CdZnTe, the Te inclusions heat up to the melting temperature, which leads to the formation of high temperature gradient field around them during the laser pulse [22].

The refraction index increases with temperature, and can be calculated by Eq. (1) presented in [23] using the temperature

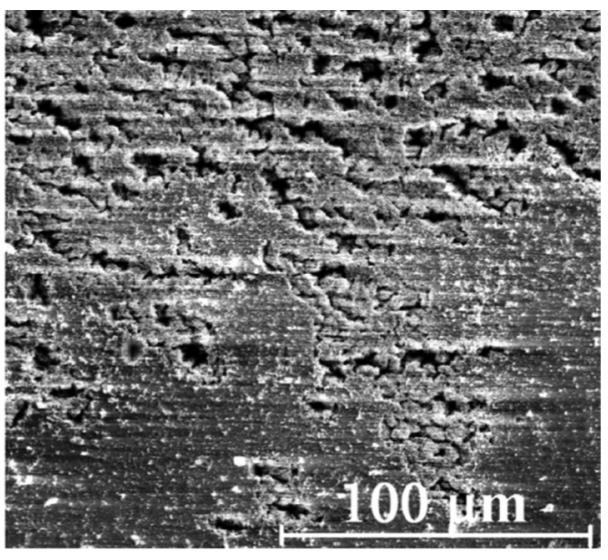

b)

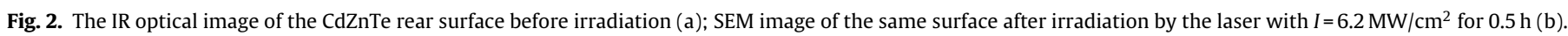



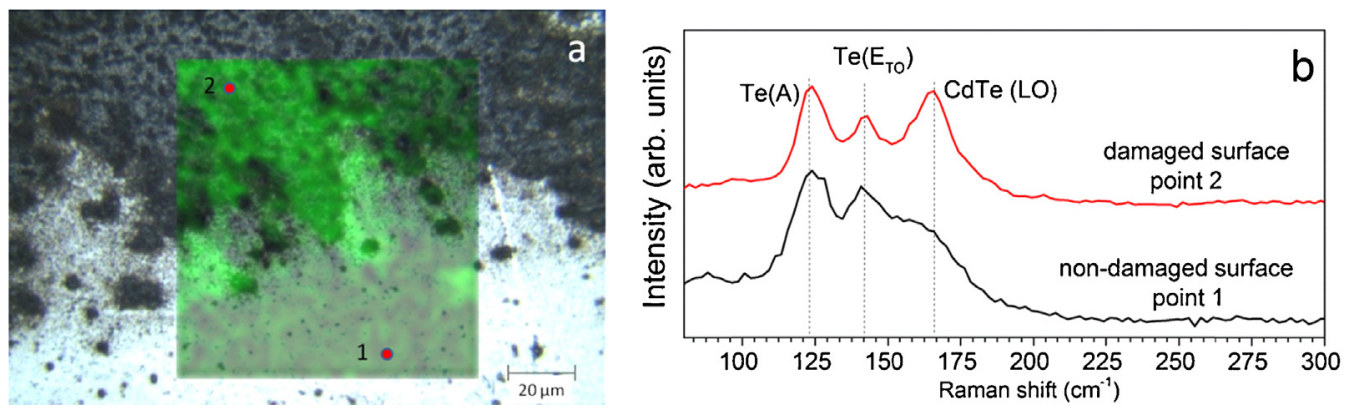

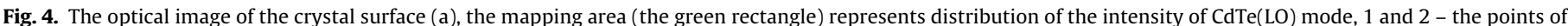
measurement of the corresponding Raman spectra (b).

coefficient of the refractive index $\left(\partial \eta / \partial T=1.5 \times 10^{-1} \mathrm{~K}^{-1}\right.$ at $1064 \mathrm{~nm})[24]$

$\Delta \eta=\left(\frac{\partial \eta}{\partial T}\right)_{p} \mathrm{~d} T+\left(\frac{\partial \eta}{\partial p}\right)_{T} \mathrm{~d} p$

where the first term is the refractive index change due to the temperature change, the second one is due to the density change.

It should be noted that $(\partial \eta / \partial T)_{p} "(\partial \eta / \partial p)_{T}$ and the second term in Eq. (1) could be neglected for CdZnTe. Thus, the calculation according to Eq. (1) shows that the increase of the temperature of the crystal to the Te melting point leads to the increase of refraction index by 0.06 . The non-uniform distribution of Te inclusions leads to the non-uniform distribution of temperature and refraction index. The changes of refraction index results in local self-focusing at inclusions and multi-focusing of laser beam.

In turn, the nonhomogeneous temperature distribution leads to the changes in refractive index and focusing of laser beam, i.e. thermal self-focusing [25].

Therefore, the intensity of the laser radiation at the rear surface increases locally. At that, the rapidly overheated Te inclusions produce high pressure normal to the surface and the following explosion associated with formation of pores takes place. In other words, the observed laser ablation is a result of the hydrodynamic expansion of Te inclusions [26], the so-called secondary phase hydrodynamic expansion.

In our case, the Te inclusions and hence the focus position of the laser beam are randomly distributed on the rear surface, therefore, the distribution of pores is inhomogeneous, too (Fig. 2b). The absence of sharp boundaries between the ablated spot and the rest of the rear surface and emerging of pores in different places after each pulse can be considered as the evidence of the abovementioned suggestion (Fig. 2b).

At the given intensity, the laser acts in multi-pulse manner, i.e. irreversible changes are accumulated from pulse to pulse, as was shown in [27], and was observed with the naked eye after $30 \mathrm{~min}$ of laser irradiation. When increasing the laser intensity to $8.5 \mathrm{MW} / \mathrm{cm}^{2}$ the damage of the front surface takes place as a result of the hydrodynamic expansion of Te inclusions, and hence the absorption of laser radiation increases resulting due to damage of the incident surface. Thus, one pulse damage of the rear surface was impossible.

A more detailed study of the damaged surface by SEM reveals microstructure of the pores (see Fig. 3).

Namely, as can be seen from Fig. 3, the walls of the pores are covered by the droplet-like species with the size varying from 40 to $300 \mathrm{~nm}$. The formation of such species could be explained by spreading of the initial material debris after explosion of the Te inclusions. The measurements with the help of the optical microscope show that the depth of the micro-pores varies from 1 to $7 \mu \mathrm{m}$. Such structuring of the surface leads to the increase of the light absorption coefficient in the visible wavelength range. Therefore, the damaged surface looks black on the optical image (Fig. 4a).

We speculate that the above-discussed processes may lead to the formation of Te-depleted material at the ablated surface. In order to verify this hypothesis, the sample was studied by the micro-Raman spectroscopy method.

In particular, to compare the phase composition of the damaged and non-damaged surfaces the center of Raman mapping area was set on their interface (Fig. 4).

The typical spectra obtained from both the surfaces at points 1 and 2 as shown in Fig. $4 \mathrm{a}$ are presented in Fig. $4 \mathrm{~b}$.

The Raman spectrum measured from point 1 on the nondamaged surface shows strong peaks of the $\operatorname{Te}(A)$ and $\operatorname{Te}\left(E_{\mathrm{TO}}\right)$ phonons at 123 and $142 \mathrm{~cm}^{-1}$ respectivetly $[18,19]$ while $\mathrm{CdTe}(\mathrm{LO})$ mode appears only as a shoulder of $\mathrm{Te}\left(E_{\mathrm{TO}}\right)$ peak at around $167 \mathrm{~cm}^{-1}$. It should be noted that such low intensity of $\mathrm{CdTe}(\mathrm{LO})$ mode relatively to the Te phonons is typical for CdZnTe Raman spectra $[18,20]$. The Raman spectrum obtained from the damaged surface (point 2) also reveals strong Te modes, but along with this, the intensive CdTe (LO) mode at $166.5 \mathrm{~cm}^{-1}$ was found. As follows from Fig. 4, the relative intensity of CdTe (LO) mode significantly increases. It can be explained by the removal of Te-inclusions during ablation. The spatial distribution of the intensity of the $\mathrm{CdTe}(\mathrm{LO})$ mode obtained from the mapping of the surface is presented in Fig. $4 \mathrm{a}$. As can be seen, $\mathrm{CdTe}(\mathrm{LO})$ mode is more intensive on the damaged surface which is consistent with the results of the single point measurements. Thus, the Raman experiment confirms our assumption about the formation of Cd-rich surface during laser ablation.

\section{Conclusion}

Two threshold intensities of laser ablation of CdZnTe crystal: 8.5 and $6.2 \mathrm{MW} / \mathrm{cm}^{2}$, for both the incident and the rear surfaces were found. We speculate that the ablation of the rear surface occurred in several steps, namely:

- heating of Te inclusions by the laser radiation;

- thermal self-focusing of the laser beam;

- hydrodynamic expansion of Te inclusions;

- formation of pores due to explosion of Te inclusions.

The ablation process was multi-impulse, i.e. the damage emerged with time delay.

Absorption of the laser radiation by the inclusions in CdZnTe crystal plays a significant role in laser-induced damage. The possible damage mechanism, associated with inclusions, is the thermal explosion due to the nonlinear heating of the inclusions, because of the self-focusing of the laser beam.

It was supposed that laser ablation leads to the removal of $\mathrm{Te}$ inclusions from the area near the rear surface, which was confirmed by Raman spectroscopy. 


\section{Acknowledgment}

This research has been financially supported by the European Social Fund within the project "Elaboration of Innovative Functional Materials and Nanomaterials for Application in Environment Control Technologies", Nr. 1DP/1.1.1.2.0/13/APIA/VIAA/30.

\section{References}

[1] T. Schlesinger, J. Toney, H. Yoon, E. Lee, B. Brunett, L. Franks, et al., Cadmium zinc telluride and its use as a nuclear radiation detector material, Mater. Sci. Eng.: R: Rep. 32 (2001) 103-189, http://dx.doi.org/10.1016/S0927796X(01)00027-4.

[2] A. Rohatgi, R. Sudharsanan, S.A. Ringel, M.H. MacDougal, Growth and process optimization of CdTe and CdZnTe polycrystalline films for high efficiency solar cells, Sol. Cells 30 (1991) 109-122, http://dx.doi.org/10.1016/03796787(91)90043-O.

[3] M. Bruder, H. Figgemeier, R. Schmitt, H. Maier, $\mathrm{Cd}_{1-y} \mathrm{Zn}_{y}$ Te substrates for $\mathrm{Hg}_{1-x} \mathrm{Cd}_{x}$ Te liquid-phase epitaxy, Mater. Sci. Eng.: B 16 (1993) 40-43, http:// dx.doi.org/10.1016/0921-5107(93)90009-C.

[4] S.M. Johnson, T.J. de Lyon, C.A. Cockrum, W.J. Hamilton, T. Tung, F.I. Gesswein, et al., Direct growth of CdZnTe/Si substrates for large-area HgCdTe infrared focal plane arrays, J. Electron. Mater. 24 (1995) 467-473, http://dx.doi.org/10. 1007/BF02657949.

[5] M. Bugar, E. Belas, R. Grill, J. Prochazka, Š. Uxa, P. Hlidek, et al., Inclusions elimination and resistivity restoration of CdTe: $\mathrm{Cl}$ crystals by two-step annealing, IEEE Trans. Nucl. Sci. 58 (2011) 1942-1948, http://dx.doi.org/10. 1109/TNS.2011.2159394.

[6] D.V. Gnatyuk, L.V. Poperenko, I.V. Yurgelevych, O.I. Dacenko, T. Aoki, Characterization of functional layers of CdTe crystals subjected different surface processing, in: 2013 IEEE Nuclear Science Symposium and Medical Imaging Conference (2013 NSS/MIC), 2013, pp. 1-4, http://dx.doi.org/10. 1109/NSSMIC.2013.6829829.

[7] A.L. Dawar, C. Jagadish, K.V. Ferdinand, A. Kumar, P.C. Mathur, Effect of laser annealing on the structural, electrical and optical properties of CdTe thin films, Appl. Surf. Sci. 22-23 (1985) 846-858, http://dx.doi.org/10.1016/0378 5963(85)90218-1.

[8] A. Medvid', D.V. Korbutyak, S.G. Krylyuk, Y.V. Kryuchenko, E.I. Kuznetsov, I.M. Kupchak, et al., Influence of powerful laser irradiation on impurity-defect structure of CdTe detector material, Nucl. Instrum. Methods Phys. Res. Sect. A 531 (2004) 157-160, http://dx.doi.org/10.1016/j.nima.2004.05.106.

[9] A. Medvid', N. Litovchenko, A. Mychko, Y. Naseka, Exciton quantum confinement in nanocones formed on a surface of CdZnTe solid solution by laser radiation, Nanoscale Res. Lett. 7 (2012) 514, http://dx.doi.org/10.1186/ 1556-276X-7-514.

[10] A. Medvid, Redistribution of point defects in the crystalline lattice of a semiconductor in an inhomogeneous temperature field, Defect Diffus. Forum (2002) 89-102, http://dx.doi.org/10.4028/www.scientific.net/DDF.210-212. 89.

[11] A. Medvid', A. Mychko, E. Dauksta, Y. Naseka, J. Crocco, E. Dieguez, The effect of laser radiation on CdZnTe radiation hardness, J. Instrum. 6 (2011) C11010, http://dx.doi.org/10.1088/1748-0221/6/11/C01101.
[12] D.K. Biegelsen (Ed.), Energy Beam-Solid Interactions and Transient Thermal Processing 1984 (MRS Proceedings), vol. 35, Cambridge University Press (April 21, 1985), Cambridge, 1984, http://www.cambridge.org/us/academic/ subjects/engineering/materials-science/energy-beam-solid-interactions-andtransient-thermal-processing-1984-volume-35\#contentsTabAnchor.

[13] A. Mychko, A. Medvid, E. Dauksta, Laser-induced increase of resistivity and improvement of optical properties of CdZnTe crystal, J. Cryst. Growth 415 (2015) 47-50, http://dx.doi.org/10.1016/j.jcrysgro.2014.12.028.

[14] I.P. Batra (Ed.), Metallization and Metal-Semiconductor Interfaces, Springer, Boston, MA, US, 1989, http://dx.doi.org/10.1007/978-1-4613-0795-2.

[15] R. Triboulet, P. Siffert, CdTe and Related Compounds; Physics, Defects, Heteroand Nano-Structures: Crystal Growth, Surfaces and Applications, Elsevier, 2009, http://dx.doi.org/10.1016/B978-0-08-046409-1.00001-0.

[16] P.S. Dobal, A. Dixit, R.S. Katiyar, Z. Yu, R. Guo, A.S. Bhalla, Micro-Raman scattering and dielectric investigations of phase transition behavior in the $\mathrm{BaTiO}_{3}-\mathrm{BaZrO}_{3}$ system, J. Appl. Phys. 89 (2001) 8085, http://dx.doi.org/10 1063/1.3991369.

[17] J. Hugmann, B.S. Richards, A. Crosky, Phase characterisation of $\mathrm{TiO}_{2}$ thin films using micro-Raman spectroscopy glancing angle X-ray diffraction, in: 2002 Conference on Optoelectronic and Microelectronic Materials Devices, COMMAD Proceedings (Cat. No. 02EX601) IEEE, 2003, pp. 181-184, http://dx doi.org/10.1109/COMMAD.2002.1237222.

[18] G.A. Kulkarni, V.G. Sathe, K.S.R.K. Rao, D.V.S. Muthu, R.K. Sharma, Micro-Raman imaging of Te precipitates in CdZnTe (Zn-4\%) crystals, J. Appl. Phys. 105 (2009) 063512, http://dx.doi.org/10.1063/1.3086274.

[19] S.A. Hawkins, E. Villa-Aleman, M.C. Duff, D.B. Hunter, A. Burger, M. Groza, et al., Light-induced tellurium enrichment on CdZnTe crystal surfaces detected by Raman spectroscopy, J. Electron. Mater. 37 (2008) 1438-1443, http://dx.doi.org/10.1007/s11664-008-0448-x.

[20] J. Min, X. Liang, J. Chen, D. Wang, H. Li, J. Zhang, Investigation of Te inclusions in CdZnTe crystalline material using Raman spectroscopy and IR techniques, Vacuum 86 (2012) 1003-1006, http://dx.doi.org/10.1016/j.vacuum.2011.11. 009.

[21] O.A. Bulgakova, N.M. Bulgakova, V.P. Zhukov, A model of nanosecond laser ablation of compound semiconductors accounting for non-congruent vaporization, Appl. Phys. A. 101 (2010) 53-59, http://dx.doi.org/10.1007/ s00339-010-5757-2.

[22] M. Meier, M.J. Harrison, S. Spalsbury, D.S. McGregor, Laser-induced thermomigration of Te precipitates in CdZnTe crystals, J. Cryst. Growth 311 (2009) 4247-4250, http://dx.doi.org/10.1016/j.jcrysgro.2009.06.017.

[23] M. Sakakura, M. Terazima, Y. Shimotsuma, K. Miura, K. Hirao, Thermal and Shock Induced Modification Inside a Silica Glass by Focused Femtosecond Laser Pulse, 2011, http://repository.kulib.kyoto-u.ac.jp/dspace/handle/2433/ 138082 (accessed 16.09.15).

[24] M. Bertolotti, V. Bogdanov, A. Ferrari, A. Jascow, N. Nazorova, A. Pikhtin, et al., Temperature dependence of the refractive index in semiconductors, J. Opt. Soc. Am. B 7 (1990) 918, http://dx.doi.org/10.1364/JOSAB.7.000918.

[25] R.W. Boyd, S.G. Lukishova, Y.R. Shen, Self-focusing: Past and Present: Fundamentals and Prospects, Springer Science \& Business Media, 2008, http:// www.springer.com/us/book/9780387321479.

[26] A. Miotello, R. Kelly, Critical assessment of thermal models for laser sputtering at high fluences, Appl. Phys. Lett. 67 (1995) 3535, http://dx.doi.org/ $10.1063 / 1.114912$

[27] A.A. Manenkov, Fundamental mechanisms of laser-induced damage in optical materials: today's state of understanding and problems, Opt. Eng. 53 (2014) 010901, http://dx.doi.org/10.1117/1.0E.53.1.010901. 